\title{
Proceedings of the XXXIth Seminar of the French- Speaking Society for Theoretical Biology
}

\author{
Eric Fanchon $\cdot$ Philippe Tracqui
}

Received: 27 January 2013/Accepted: 29 January 2013/Published online: 24 February 2013

(C) Springer Science+Business Media Dordrecht 2013

The articles of the present special issue were presented at the XXXIst Seminar of the French-Speaking Society for Theoretical Biology (Société Francophone de Biologie Théorique, SFBT) held in Autrans (France) from 15 to 18 may 2011.

As is the case for most scientific disciplines, the frontiers are moving. New experimental approaches are constantly being developed and older ones are pushed to new limits. Some terms are gaining wider acceptance, like Systems Biology or Complex systems science. But of course the domains covered by these terms are largely overlapping with Theoretical Biology. Biological systems are complex systems, along with social, economic, urban systems and many others. Theoretical ideas developed in one field can often be applied in another. The idea of regulation mechanism and regulatory loop has been put into practice by engineers for centuries (e.g. the famous centrifugal governor used in windmills since the seventeenth century, and rediscovered by Boulton and Watt), applied in physiology (Claude Bernard defined homeostasis in 1865), and theorized in the twentieth century by cyberneticians. It has become since then a pervasive concept with applications in many disciplines. This is a striking example of a powerful theoretical idea which provides a unifying frame. Systems Biology, for its part, has grown with the dissemination of high-throughput technologies (DNA and RNA sequencing, proteomics, interactomics, etc.) and is perceived by many as the sum of these technologies and associated data-processing tools. But the mechanisms at work within cells, organisms and populations can only be understood by building and analyzing multi-scale models, and modeling has indeed become an integral part of Systems Biology.

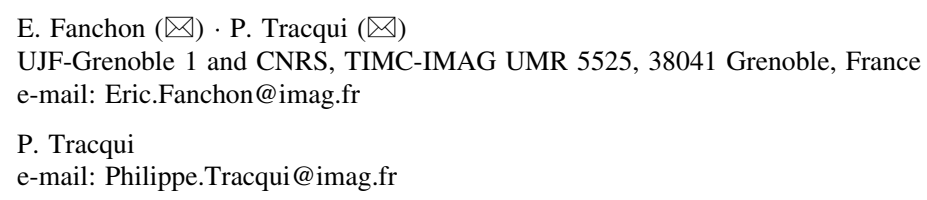


For the 2011 edition of the seminar the following leading theme was proposed: multi-scale regulation networks and biological signal transduction (Réseaux de Régulation Multi-échelles et Transduction de Signaux Biologiques). Several sessions were devoted to it, beside general sessions. High level invited lectures provided up-to-date introductions to the seminar themes.

The present issue gives a selected and representative sampling of the problems that are debated at SFBT seminars and conferences: multiscale modeling (LahutteAuboin et al.; Lesne), intra-cellular networks (Ben Amor et al., Ndiaye and Gouzé, Sepulchre and Ventura), physiology and medicine (Lahutte-Auboin et al.; Le Rolle et al.), population dynamics (Bensenane et al.; Rault et al.), epidemiology (Demongeot et al.).

The SFBT encourages cross-disciplinary exchanges and the circulation of ideas. We were particularly pleased to see that many young scientists attended this XXXIth seminar. Scientific awards were attributed to the most distinguished young researchers: the Pierre Delattre prize to Geoffroy Andrieux (GERHM, Rennes) and to Anthony Malkassian (MIO, Marseille), and the prize of the best poster to AnneCécile Lesart (TIMC-IMAG, Grenoble).

Acknowledgments The SFBT wishes to thank The Rhône-Alpes Complex Systems Institute (IXXI), Université Joseph Fourier, and Centre National de la Recherche Scientifique (CNRS) for financial support. 